\title{
El Proceso de Desarrollo de Productos Tecnológicos entre las Universidades y las MIPYMES Mexicanas: Una Carrera de Obstáculos
}

\author{
Luís Roberto Vega González'
}

\begin{abstract}
Today firm's competitiveness depends on the development of proprietary technology and its integration to commercial technological products. Experiences and case studies show that the micro, small and medium firms (MIPYMES, in Spanish) develop technological products collaborating with public universities. In this work it is presented the colaborative technological product development process phases focusing in the identification of the diverse obstacles occurring along the process. Diverse technical, organizational and communication aspects that affects the project development are discussed. The resistances or barriers to the development make it difficult and could cause project abandon sometimes. This empirical and exploratory research is conducted under the reference of the technological project management model developed and used at the Centro de Ciencias Aplicadas y Desarrollo Tecnológico (CCADET) of the Universidad Nacional Autónoma de México (UNAM).
\end{abstract}

Keywords: technological development barriers; resistance; technological products; Small \& Medium enterprises.

\section{Resumen}

Hoy en día se sabe que la competitividad de las empresas depende del desarrollo de tecnologías propietarias y de su integración a productos tecnológicos comercializables. Muchas experiencias y estudios de caso demuestran que las MIPYMES ${ }^{2}$ desarrollan productos tecnológicos colaborando con las universidades públicas. En este trabajo se presenta una revisión de las fases del proceso de desarrollo de productos tecnológicos colaborativo poniendo énfasis en la identificación de los diversos obstáculos que se presentan a lo largo del mismo. Se discuten diversos aspectos técnicos, organizacionales y de comunicación que afectan al proceso convirtiéndose en resistencias o barreras que lo obstaculizan y que pueden llegar inclusive a provocar su abandono. Esta investigación empírica y exploratoria se enfoca desde la perspectiva del modelo de Gestión Tecnológica de Proyectos (GTP) que se lleva a cabo en el Centro de Ciencias Aplicadas y Desarrollo tecnológico, CCADET de la Universidad Nacional Autónoma de México, UNAM.

Palabras Claves: barreras o resistencias al desarrollo; productos tecnológicos; MIPYMES.

\footnotetext{
I Centro de Ciencias Aplicadas y Desarrollo Tecnológico, CCADET-UNAM. Coordinación de Vinculación y Gestión Tecnológica. Tels. 5622-8602 ext. II 35 y II 85 ; Fax 5622-8626, Irvg@servidor.unam.mx; roberto.vega@ccadet.unam.mx

2 Empresas micro, pequeñas y medianas.
} 


\section{Introducción}

Sabemos que el hecho de desarrollar y administrar tecnología en forma adecuada es lo que crea riqueza para las naciones, compañías e individuos, por lo tanto es el fundamento del crecimiento y determina la competitividad nacional y organizacional, particularmente en un mercado global y fieramente competitivo. (Khalil y Ezzat, 2005).

Kondo, (2005) propone que para el desarrollo económico de Japón ha sido crucial un proceso de adquisición y transferencia de tecnología del exterior, aunado a un proceso de asimilación y escalamiento interior, para que el país posteriormente estuviera en condiciones de construir sus propias capacidades internas de desarrollo. De ésta forma, la tecnología se ha convertido en una máquina para el crecimiento económico. Por lo tanto, el progreso tecnológico es la clave de la competitividad internacional y del crecimiento económico interno. (Kondo, op.cit. 2005, p. 156). La pregunta clave es: ¿si en los países en vías de desarrollo sabemos que se requiere desarrollar tecnología, por qué no lo hacemos? En este trabajo trataremos de encontrar algunas respuestas.

En todos los países se desarrollan prototipos tecnológicos de distinto nivel en las organizaciones públicas y privadas que operan en los diferentes sectores económicos y sociales, incluyendo a las universidades y las instituciones de investigación y desarrollo; sin embargo, la incorporación de los prototipos al sistema productivo ya sea para desarrollar nuevos productos o mejorar las características de los productos existentes en la forma de innovaciones incrementales, es un trabajo que corresponde a las empresas.

En los países desarrollados y en vías de desarrollo, las empresas pequeñas y medianas (PYMES) son reconocidas como el pilar de la economía. Por ejemplo, según Villanueva, (2009), en Madrid España las PYMES han sido responsables del $80 \%$ de los empleos creados en los últimos cuatro años, mientras que en México con base en información del Instituto de Estadística, Geografía e Informática (INEGI), se estima que las micro, pequeñas y medianas empresa (MIPYMES) constituyen el $99 \%$ del total de las Unidades económicas del país generando más del 50\% del Producto Interno Bruto. (Diario Oficial, 2008).
Así pues, el desarrollo de productos tecnológicos a partir de innovaciones tecnológicas radicales es una situación que solo se da en algunos casos aislados en las MIPYMES por lo que queda fuera del alcance de este trabajo.

\section{El proceso de desarrollo de productos tecnológicos}

En la Figura I se muestran las etapas del proceso de desarrollo de productos tecnológicos. Las etapas D0 a D4 se identifican en el esquema como etapas de desarrollo de prototipos tecnológicos o tecnologías precompetitivas; mientras que las etapas de mercado se identifican como MO y MI.

El proceso inicia en Do frecuentemente a partir de las ideas que surgen de la investigación básica, tratando de dar respuesta a alguna demanda detectada en el mercado. Otras veces se trata de proyectos contratados por empresas a los Institutos y Centros de I\&DT. Las MIPYMES Mexicanas carecen de recursos económicos y del capital intelectual necesario para el desarrollo de tecnología por lo que casi siempre recurren al establecimiento de alianzas con Instituciones de I\&DT públicas y buscan apoyo de las entidades de fomento gubernamentales para el financiamiento de los proyectos.

En la primera etapa (D0 a DI), con una inversión mínima se inicia la construcción de un dispositivo preliminar o prototipo de banco que sirve para realizar la valoración inicial de las posibilidades del desarrollo tecnológico. En este prototipo preliminar las tarjetas electrónicas, los ensambles mecánicos, ópticos, acústicos y de sensores se soportan en cajas o cubiertas improvisadas y se interconectan con montones de alambres. A veces las entradas y salidas no tienen terminales atornilladas sino que se realizan conexiones con alambres trenzados en forma provisional. Los despliegues son tomados en préstamo de otros experimentos y por lo tanto el sistema en su conjunto no tiene una gran exactitud ni precisión. Entre las etapas DI a D2 se realizan mejoras al prototipo de banco hasta llegar a un prototipo de concepto mediante el cual se logra la validación de la factibilidad técnica y funcional del dispositivo. 


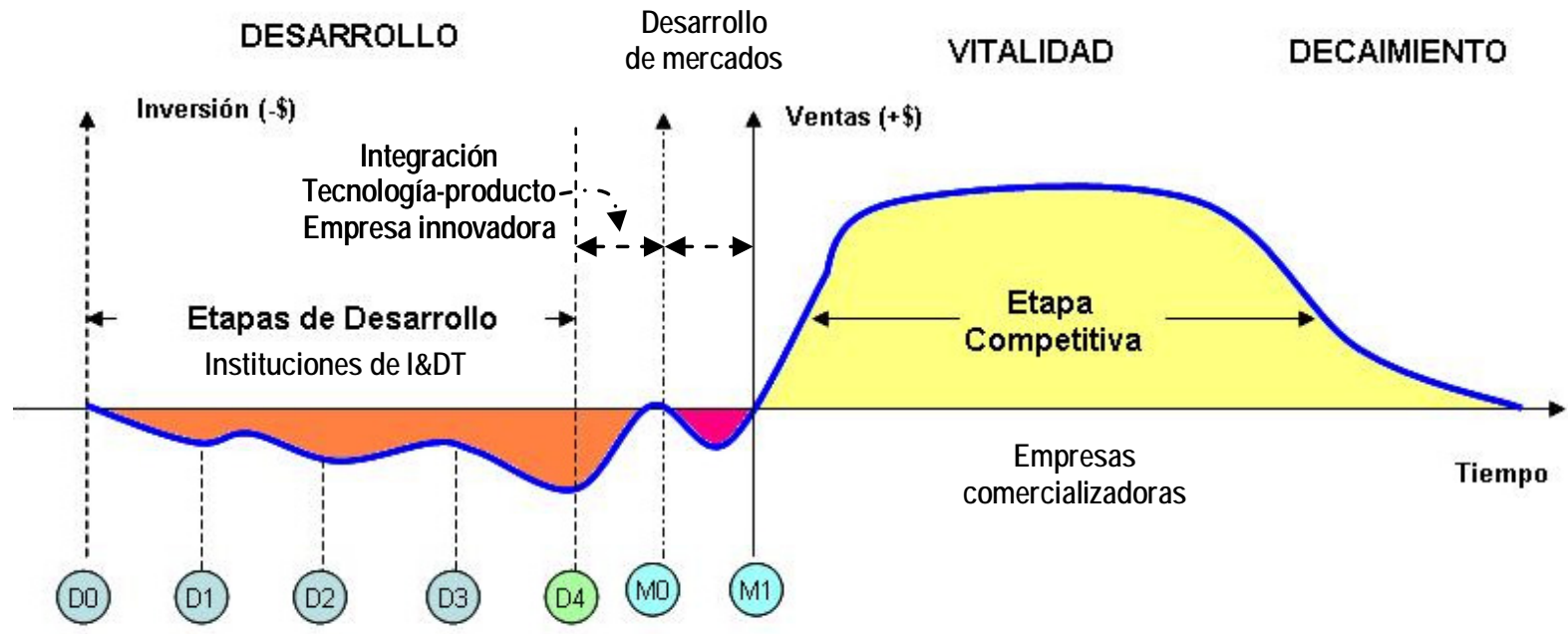

Figura I. Etapas en el proceso de desarrollo de productos tecnológicos

El prototipo de concepto consume una mayor cantidad de recursos en la forma de materiales, equipo de prueba y de medición, así como de capital intelectual en la forma de salarios del personal académico y técnico, administración y un porcentaje de indirectos asociado al proyecto. Casi siempre los prototipos de concepto son artesanales. Muchas Oficinas de Transferencia de Tecnología universitarias a nivel internacional como parte de sus funciones proveen financiamiento para las pruebas de concepto. (Franzoni 2007; Biotechnology Ireland, 2009).

En al etapa D2 a D3 se realizan intensas actividades de mejora que consumen una gran cantidad de recursos. Los grupos científicos ofrecen diferentes montajes experimentales de laboratorio y el personal técnico trabaja intensamente para obtener una versión mejorada del prototipo de concepto preliminar. Para cada mejora se requiere mucha iteración en los prototipos de desarrollo. Actividades como el ensamble, calibración, pruebas, diseños de ingeniería y modificaciones se realizan varias veces antes de terminar una nueva versión del prototipo. Dependiendo de la complejidad del proyecto este proceso puede tomar bastante tiempo y requiere de la disponibilidad de materiales y recursos.

El equipo de proyecto también debe trabajar en nuevas características y especificaciones, además el proyecto se extiende hacia los grupos de ingeniería y diseño, los cuales a su vez deben trabajar en aspectos como la usabilidad, la confiabilidad y la disponibilidad del dispositivo tecnológico. También se trabaja en el aspecto exterior mejorando las cajas, los contenedores, los despliegues y la apariencia global del dispositivo tecnológico. Al final de la etapa D3 se obtiene un prototipo de laboratorio que ya tiene el carácter de tecnología pre-competitiva.

Muchas veces aquí termina la intervención de los grupos académicos universitarios que realizan I\&DT en los proyectos de innovación. En este punto se procede a obtener los certificados o títulos de propiedad intelectual que puedan resultar del prototipo tecnológico obtenido en el proyecto de desarrollo. Cuando los prototipos fueron desarrollados con financiamiento externo, inmediatamente se procede son los trabajos de transferencia de tecnología (TT) de los derechos de propiedad intelectual y la negociación de sus términos. Generalmente se requiere de una corrida de valuación tecnológica para definir los términos de los contratos de TT. Una vez que se realiza la transacción, la tecnología pasa a ser un activo tangible para las instituciones de I\&D públicos. La TT se realiza en algún momento entre las etapas D3 y D4. La fase de transferencia que se da entre D4 y M0 es crucial ya que la empresa receptora debe asimilar, escalar e integrar la tecnología que recibe a sus propios procesos productivos para obtener un prototipo piloto preindustrial del producto tecnológico. En la etapa pre comercial (M0 a MI) se requiere nuevamente de fuertes inversiones para generar y poner en marcha las estrategias de marketing

ISSN: 07I 8-2724. (http://www.jotmi.org)

Journal of Technology Management \& Innovation ( Universidad Alberto Hurtado, Facultad de Economía y Negocios 
por las empresas manufactureras innovadoras. Después de la etapa MI inician las ventas y la difusión en el mercado del producto tecnológico con lo que finalmente se espera un flujo de recursos positivo $(+\$)$ por las ganancias que se espera sean generadas de las ventas de la tecnología que se difundirá en el mercado.

\section{Diferentes tipos de obstáculos en el desarrollo de productos tecnológicos}

Para identificar los diferentes tipos de resistencias $\mathrm{u}$ obstáculos que se presentan en el proceso de desarrollo de productos tecnológicos en la Figura 2 se presenta una ampliación de la Figura I. Los tipos de resistencias detectadas son: (a) las resistencias relativas a aspectos financieros $\left(R_{F s}^{\prime}\right)$, (b) las resistencias relativas a aspectos de organizacionales o humanos $\left(R_{O}^{\prime}\right)$, (c) las resistencias relativas a aspectos de índole técnico o de gestión tecnológica $\mathrm{u}$ organizacional $\left(R_{D T s}{ }^{\prime}\right)$, las resistencias relativas a aspectos jurídicos $\left(R_{j s}^{\prime}\right)$ y las resistencias relativas a aspectos de mercado $\left(R_{M s}^{\prime}\right)$. 


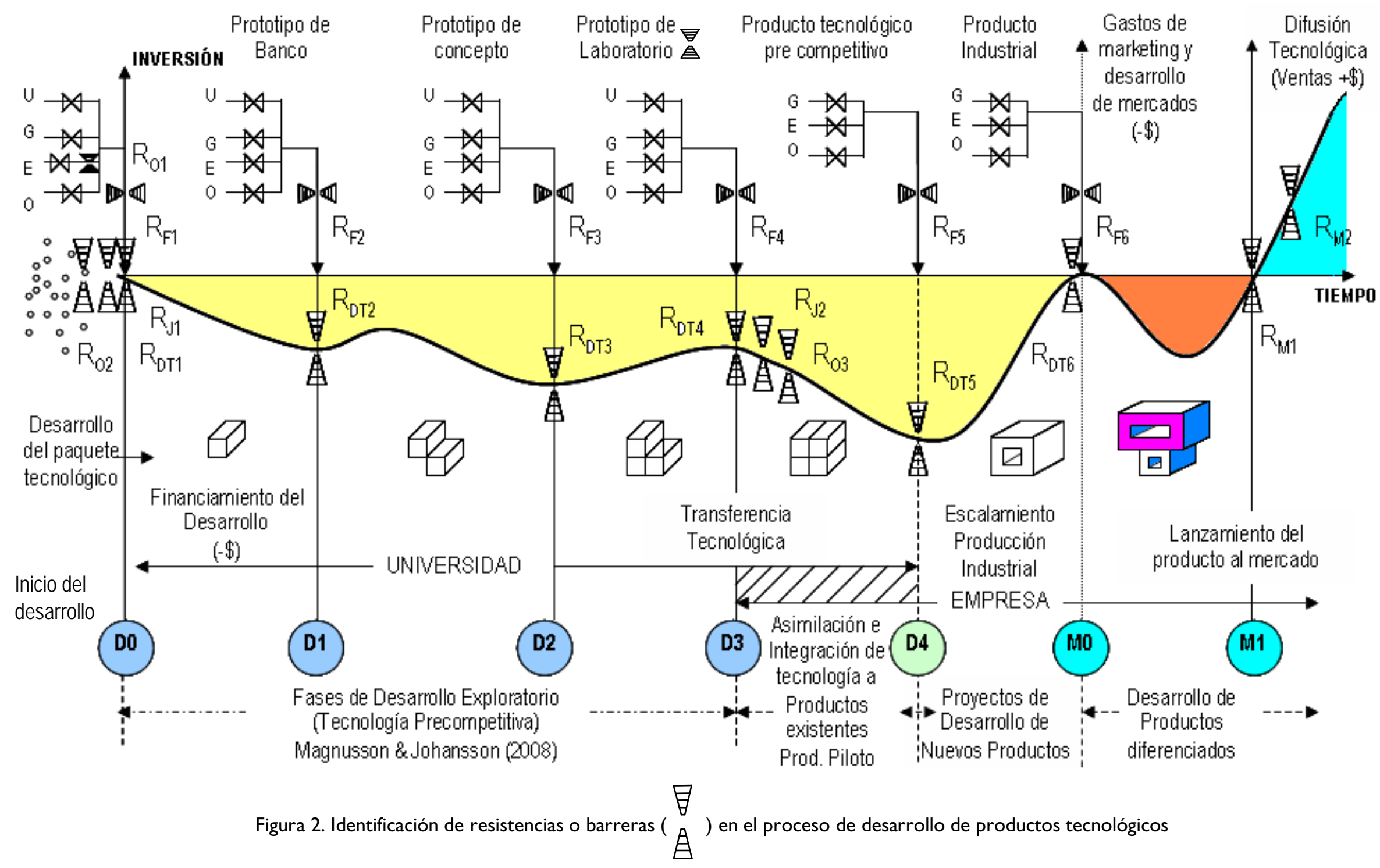




\section{El desarrollo colaborativo del producto tecnológico: la gran carrera de obstáculos}

La intensa competencia global de la actualidad determina la aparición en el mercado de productos con ciclos de vida cada vez más cortos. Por esta razón las grandes empresas definen dentro de su organización unidades de planificación y gestión del ciclo de vida de sus productos. Según Cruz y Martínez (2009), el ciclo de vida es dinámico y depende del cambio tecnológico y de los cambios en las preferencias de los consumidores, esto es un factor que contribuye a que el proceso de desarrollo de productos tecnológicos se convierta en una verdadera carrera de obstáculos.

La carrera inicia en D0 una vez que la empresa, después de realizar un estudio de factibilidad técnica y de mercado, se convence de que debe desarrollar tecnología en lugar de comprarla, esto implica salvar la resistencia organizacional $\boldsymbol{R}_{\mathrm{OI}}$. Si se trata de una MIPYME normalmente buscará tener contacto con una universidad o una institución pública de I\&DT para plantearle su demanda específica de desarrollo de tecnología. A través de diversas reuniones entre los equipos de trabajo se discuten $y$ definen las primeras especificaciones del proyecto. La institución de I\&DT define su equipo de trabajo normalmente con académicos $y / o$ personal científico y técnico con buena disposición para participar en este tipo de proyectos. Con esto se salva $\boldsymbol{R}_{\mathbf{O} 2}$. Para salvar $\boldsymbol{R}_{D T I}$ la institución de I\&DT propone y acuerda con la empresa una vía de trabajo de entre múltiples opciones para desarrollar un primer prototipo de banco de bajo costo. Este prototipo servirá para evaluar la factibilidad técnica de cumplir con las especificaciones preliminares. Algunas veces la empresa puede financiar esta parte del desarrollo con sus propios recursos económicos. Otra posibilidad es que la misma universidad o institución de I\&DT aporte recursos para esta primera prueba de factibilidad de bajo costo $\circ$ bien que la empresa haya obtenido recursos de alguna institución de fomento o de alguna otra fuente alterna. Con cualquiera de estas alternativas se salva $\boldsymbol{R}_{F I}$.

Con el consenso de la empresa, la institución de I\&DT y de la institución de fomento, finalmente se acuerda la firma de un Convenio Colaboración para el desarrollo conjunto de la tecnología. En este momento inicia la participación de los grupos jurídicos de las tres partes. Normalmente llegar a la firma del instrumento jurídico es bastante complicado por lo que cuando se llega al momento en el que se acuerdan todos los términos jurídicos se salva la primera barrera jurídica $\boldsymbol{R}_{\jmath \prime}$. El convenio de desarrollo típicamente cubre el tiempo y el financiamiento requeridos para el desarrollo de los prototipos de banco (DI) y del prototipo de concepto (D2), en algunos casos inclusive se cubre inclusive hasta el desarrollo del prototipo de laboratorio (D3).

A partir de la firma del convenio de desarrollo inician los trabajos de acuerdo con el calendario aprobado para el proyecto. Un prototipo de banco normalmente tomará solo unos cuantos meses, dependiendo del tipo de tecnología que se desarrollará.

Al llegar la fase DI se confirmará la factibilidad técnica de obtener el prototipo de banco. Con esto se salvará el obstáculo $\boldsymbol{R}_{\mathrm{DT2} \text {. }}$ Si el prototipo de la tecnología de banco es aprobado por la empresa entonces se aprobará la entrega de la segunda aportación de recursos con lo que se salvará la segunda resistencia financiera $\boldsymbol{R}_{\mathbf{F} 2}$.

Con los recursos económicos requeridos para la compra de materiales e insumos continúan los trabajos para desarrollar el prototipo de concepto de la fase D2.

Si se logra el desarrollo del prototipo de concepto se aprueba la factibilidad técnica con lo que se salva el

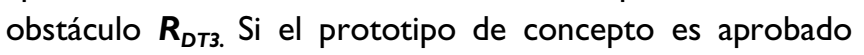
por la empresa entonces se aprobará la entrega de la tercera aportación de recursos con lo que se salvará la tercera resistencia financiera $\boldsymbol{R}_{\mathbf{F} 3 \text {. }}$

De forma similar se salvan las resistencias $\boldsymbol{R}_{D T 3}$ y $\boldsymbol{R}_{F 3}$ al llegar a la fase D3 con el desarrollo y aprobación del prototipo de laboratorio. Como dijimos previamente, en la mayoría de los casos el convenio de desarrollo inicial cubre hasta esta fase del proyecto.

La Tabla I muestra los distintos equipos de trabajo que tienen que colaborar para sortear las barreras que se presentan en las distintas fases del desarrollo de productos tecnológicos.

$\mathrm{Si}$ el prototipo de laboratorio es aprobado, se salva la barrera $\boldsymbol{R}_{\mathrm{DT} 4}$ y llega el momento de definir la estrategia de protección más adecuada para los desarrollos. Aquí deberá definirse si es posible obtener patentes, modelos de autor, diseños industriales, derechos de autor, o una mezcla de estos. Los derechos de explotación de la propiedad intelectual de la tecnología serán el objeto del nuevo 
convenio de licenciamiento o transferencia de tecnología. Para definir los términos de cambio; es decir, la forma de pago de la tecnología en alguna de sus modalidades, regalías, pago único o esquemas mixtos será necesario realizar un ejercicio de avalúo tecnológico. Normalmente cuando se aprueban los términos de pago y el paquete tecnológico que se transferirá, se procede con la firma del convenio de transferencia de tecnología para lo cual intervendrán los jurídicos de la empresa y de la institución de I\&DT. En este momento se salvara la barrera jurídica $\boldsymbol{R}_{\mathbf{j} 2}$.

La empresa deberá nuevamente usar recursos propios para la fase de transferencia, asimilación e integración de la tecnología desarrollada para mejorar algún producto ya existente o para desarrollar algún nuevo producto.

\begin{tabular}{|l|l|}
\hline \multicolumn{1}{|c|}{ Fase } & \multicolumn{1}{c|}{ Equipo de trabajo } \\
\hline (D0) & $\begin{array}{l}\text { Firma de convenios de DT } \\
\text { Equipo jurídico de la empresa+ equipo jurídico de la institución de I\&DT + jurídico de la } \\
\text { institución de fomento patrocinadora+personal de vinculación y gestión tecnológica (V\&GT) }\end{array}$ \\
\hline (D0 a D3) & $\begin{array}{l}\text { Desarrollo exploratorio de prototipos tecnológicos } \\
\text { Responsables de la empresa+equipo de I\&DT+ personal de gestión tecnológica }\end{array}$ \\
\hline (D3) & $\begin{array}{l}\text { Transferencia de Tecnología } \\
\text { Jurídico empresa +l\&DT+personal V\&GT } \\
\text { Equimilación y adaptación de prototipos al producto }\end{array}$ \\
\hline (D4 a M0) & $\begin{array}{l}\text { Escalamiento industrial y desarrollo de producto prototipo } \\
\text { Equipo técnico de la empresa }\end{array}$ \\
\hline (M0 a MI) & $\begin{array}{l}\text { Lanzamiento del nuevo producto y estrategia de marketing } \\
\text { Equipos de marketing y técnico de la empresa }\end{array}$ \\
\hline
\end{tabular}

Tabla I. Equipos de trabajo requeridos para el desarrollo de nuevos productos tecnológicos

También es posible que la empresa busque recursos de alguna institución de fomento o de alguna otra fuente. Cuando esto se logra se salva la resistencia financiera $\boldsymbol{R}_{\mathbf{F 4}}$. Para continuar con el desarrollo del producto piloto, existen muchos problemas organizacionales para definir los equipos de desarrollo pruebas y calidad del producto mejorado. En principio se requiere de ciertas capacidades tecnológicas y administrativas ya que según Mostert y Buys (2008), para eliminar las barreras en la transferencia se requiere de la aplicación de puentes de tipo general, facilidades de prueba y soporte para usuarios, el desarrollo de sociedades y alianzas, liderazgo y la disposición de explorar y aprender así como de desarrollar procedimientos de comunicación entre los miembros del equipo de trabajo. Solo cuando la empresa es capaz de librar estos obstáculos se tiende el puente para sobrepasar el obstáculo organizacional $\boldsymbol{R}_{\text {Oз }}$.
Después de la firma del convenio inicia la primera transferencia de tecnología real con la entrega del paquete tecnológico por parte de la institución I\&DT al equipo de desarrollo de la empresa. Por lo tanto, la asimilación e integración de tecnología se da en las instalaciones de la empresa.

Durante todo este proceso, que sucede entre las fases D3 a D4, el equipo de trabajo de la institución de I\&DT participa en forma intensiva con el equipo de la empresa a través de asesorías y consultorías, hasta lograr la realización del producto piloto mejorado con lo cual se rompe la barrera $\boldsymbol{R}_{\mathrm{DTS}}$.

En la fase D4 se da una nueva transferencia interna en empresas medianas y grandes; es decir, el producto piloto se transfiere del equipo de desarrollo de nuevos productos al equipo de manufactura y producción. (Jing y Zhou, 2008). 
En aquellos casos en los que la empresa contrató el desarrollo de tecnología con alguna entidad internacional, la transferencia de conocimiento se dificulta aún más por las diferencias nacionales en las tradiciones científicas y los modelos culturales que dificultan la entrega de tecnología apropiada a la empresa contratante. (Vera y Reigota, 2007).

Cabe aclarar que en una MIPYME el mismo equipo de trabajo desarrolla las actividades de I\&D y el desarrollo de nuevos productos, por lo tanto no existe transferencia de conocimiento entre grupos. Las cosas se complica aún más, porque para salvar la barrera $\boldsymbol{R}_{\mathrm{F} 5}$, la empresa requiere de la aplicación de nuevos recursos económicos ya sea propios o bien del patrocinio de alguna institución de fomento al desarrollo o de la participación económica de nuevos socios interesados en el proyecto. Invirtiendo los recursos financieros y el talento necesario, solo es hasta que se demuestra la factibilidad técnica de la fabricación industrial plena del producto, que se salva la resistencia técnica $\boldsymbol{R}_{\mathrm{DT}}$.

Para las fases del lanzamiento del nuevo producto tecnológico que se dan entre $\mathrm{MO}$ a $\mathrm{MI}$, nuevamente la empresa requerirá de la disposición de suficientes recursos económicos para fabricar los primeros lotes de producción y para desarrollar y poner en operación las estrategias de marketing, publicidad, ventas y actualización del estudio de mercado. Los recursos podrán ser propios, de instituciones de fomento o de otros aliados y socios interesados. Con estos recursos se puede brincar la barrera $\boldsymbol{R}_{\mathrm{F} 6}$.

Después de todos los obstáculos económicos, organizacionales y técnicos por los que ha pasado el proyecto, es en MI cuando se presenta la verdadera prueba de fuego para el estudio de mercado que se hizo al principio del proyecto y sus diferentes actualizaciones. Una vez que el nuevo producto se encuentra en el mercado habrá que ver ¿cómo será recibido y percibido en el mercado?, ¿será aceptado?, ifuncionará la campaña de marketing? la gente, las empresas, las organizaciones a las que esta dirigido ¿lo comprarán?, ¿durante cuánto tiempo? ¿Cuál será el tiempo de vitalidad del producto de acuerdo a su ciclo de vida?, entre muchas otras. Con las primeras ventas se iniciará el flujo económico positivo que aplicará en primera instancia al retorno de la inversión. Con esto el equipo conductor del proyecto por parte de la empresa podrá brincar el obstáculo $\boldsymbol{R}_{M I}$ y evaluar la necesidad de instalar una nueva línea de producción continua.
Finalmente, una vez que el nuevo producto se posiciona en el mercado a través de una buena percepción en el público consumidor quien medirá y comparará sus atributos de funcionamiento, usabilidad, confiabilidad, calidad y precio, para que el nivel de ventas siga creciendo es probable que deba de romper un último obstáculo al competir con algunos intereses monopólicos que probablemente ya se encuentren posicionados y sean los dominantes en el mercado. Si los estrategas de la empresa en el campo de las ventas, la negociación y las licitaciones públicas son capaces de sobreponerse a ese dominio, entonces la empresa vencerá el obstáculo $\boldsymbol{R}_{M 2}$ y continuará con la difusión del producto tecnológico en el mercado. En este momento el proyecto del desarrollo de un nuevo producto tecnológico se convierte en una verdadera innovación exitosa.

Hasta aquí se han descrito las dificultades que hay que salvar a lo largo de todo un proyecto global que inicia con la idea y la concepción del proyecto de desarrollo de tecnología, hasta que se realiza la innovación en el mercado con su correspondiente impacto social. Sin embargo, hay que aclarar que pueden existir proyectos parciales que incluyan una sola fase, dos, etcétera. Por ejemplo, en muchas universidades se desarrollan prototipos de banco y de concepto con recursos propios para fines académicos tales como la demostración de principios científicos y la realización de tesis de licenciatura, maestría y doctorado.

Las empresas casi nunca presentan interés por los prototipos académicos, por lo que los mismos solamente se reportan en los informes y publicaciones respectivas y sus partes y materiales se utilizan en nuevos prototipos. Otras veces las empresas identifican las capacidades de las instituciones de I\&DT por medio de su monitoreo tecnológico y se interesan en cierto conocimiento reportado. Entonces se puede iniciar un proyecto en la fase D2 para desarrollar la tecnología a nivel laboratorio. Si hay buenos resultados, el proceso puede continuar hasta el lanzamiento del producto.

En el caso de empresas medianas y grandes hay otros proyectos en los que el grupo de I\&DT de la firma desarrolla su propia tecnología para nuevos productos 0 para mejorar las características de productos existentes. En estos proyectos que se realizan entre las fases D3 a D4 la responsabilidad del desarrollo recae en el equipo de la empresa pero en algunos casos puede contar con asesoría de científicos y técnicos de universidades públicas. Hay 
casos en que las universidades apoyan a las empresas en las fases de lanzamiento de producto M0 a MI por medio de asesores en marketing, negociaciones y ventas.

\section{Conclusiones}

No es fácil desarrollar tecnología a través de la colaboración de las MIPYMES y las universidades, ni integrarla a productos nuevos o mejorados, hay que vencer una cantidad enorme de barreras técnicas, organizacionales, jurídicas y de mercado, para esto, se requiere de la construcción de puentes de comunicación y gestión que permitan la obtención de recursos suficientes, la transferencia efectiva de conocimiento, la formación de alianzas y sociedades con la capacidad de transmitir y recibir información, con una buena disposición para comunicarse. (Mostert y Buys, 2008).

El desarrollo de tecnología es de alto riesgo, ya que existen múltiples problemas de índole técnico, hemos observado que tiene frente borroso (fuzzy front end) identficado como $\boldsymbol{R}_{D T I}$ y final borroso $\boldsymbol{R}_{D T S}$ (fuzzy end). De hecho, podríamos decir que todo el proceso de desarrollo es borroso; es decir, no se ve muy claro el camino.

Para que una MIPYME se pueda subir a la corriente de desarrollo de un nuevo producto tecnológico requiere de grandes cantidades de capital para I\&DT, que se aplicarán en equipos, infraestructura, instituciones de I\&DT, salarios del capital humano calificado e innovador y de los lideres que administrarán el proceso de sintonización organizacional requerido para llevar a cabo los objetivos del proyecto. También requiere de buenos estrategas de mercado y de los materiales y equipos específicos para su aplicación en los trabajos propios del proyecto y sus pruebas de calidad y normalización. Naturalmente para llevar a cabo la articulación entre los grupos de trabajo, la gestión jurídica, el manejo de propuestas técnico económicas y convenios, la gestión financiera, la estrategia de propiedad intelectual, la gestión de pruebas de calidad y la evaluación de la conformidad y la seguridad, se requiere de la participación continua de un equipo de Gestión Tecnológica.

Por lo tanto concluimos enfáticamente que el desarrollo de nuevos productos tecnológicos es complejo y según Hughes y Chafin (1996), debe considerarse como un proceso de aprendizaje continuo. Las universidades deberían considerar como parte de su misión ya no ser solamente proveedoras de educación y nuevo conocimiento en la forma de publicaciones, sino también mantenerse pendientes de las necesidades de la sociedad y del mercado y realizar esfuerzos intensos para vincularse con las empresas y apoyarlas en las distintas tareas que demanda la gestión de nuevos proyectos tecnológicos. (Franzoni, 2007). Las universidades pueden participar a lo largo de todo el proceso o con actividades parciales que van desde los servicios de consultoría y de asesoría, el desarrollo de prototipos tecnológicos, hasta el acompañamiento para el lanzamiento de los nuevos productos a través de la definición de estrategias de propiedad intelectual y de mercado. No solo deben formar recursos humanos, sino que también deben desarrollar emprendedores y equipos de trabajo con actitud positiva hacia la innovación. Cada nuevo producto que se lanza al mercado o cada innovación incremental en los productos existentes impulsarán la economía y tendrá algún impacto en la mejora del índice de calidad de vida de la sociedad. Por supuesto que al mismo tiempo se generarán conocimiento y competencias de segundo orden; es decir, al recorrer el camino aprenderemos a aprender $y$ a colaborar formando equipos de trabajo verdaderamente productivos. La apuesta es que mediante este proceso las MYPIMES de los países en vías de desarrollo podrán insertarse competitivamente en la economía global. En México desarrollar este tipo de proyectos ya no es opcional, es mandatario.

\section{Referencias}

BIOTECHNOLOGY IRELAND (2009). Commercializing Biotech, Enterprise Ireland. Disponible en http://www.biotechnologyireland.com/bfora/systems/xmlvie wer/default.asp?arg=DS_BIR_NOTEART_I_list.xsI/2.

[Consultado el 3 de agosto de 2009].

CRUZ, R., Martínez, C. (2009). Tema 8: Estrategias competitivas dinámicas; Dirección Comercial II, Universidad Autónoma Metropolitana, disponible en http://www.uam.es/personal_pdi/economicas/javierg/docenc ia/Derecho/Tema\%208.doc; [Consultado el 30 de abril de 2009].

DIARIO OFICIAL DE LA FEDERACIÓN (2008). Acuerdo por el que se establecen las Reglas de Operación para el otorgamiento de Apoyos del Fondo de Apoyo para la Micro, Pequeña y Mediana Empresa (Fondo PyME). $15^{\circ}$ Sección; Secretaría de Economía, 29 de diciembre. 
FRANZONI, Ch. (2007). Opportunity recognition in technology transfer organizations: five case studies from UK and Italy. International Entrepreneur Management Journal. Vol 3, pp. 5I-67

HUGHES D., Chafin D. (1996). Product Development Continuous Learning Process, Journal of Product Innovation Management, Vol. 13, Pp. 89-104. Elsevier Science Ltd., New York, USA.

JING Z. J., Zhou, Y. T. (2008). Knowledge integration based technology transfer mode for manufacturing firms, IEEE Chinese Control and Decision Conference (CCDC), pp.44804886.

KHALIL, T., Ezzat, H. (2005). Management of Technology and responsive policies in new economy, International Journal of Technology Management (IJTM), Vol. 32 Nos. I/2; PP. 88-III.

KONDO, M. (2005). Networking for technology Acquisition and transfer. International Journal for Technology Management, Vol. 32, No's 1/2.

MOSTERT J. N., Buys A. J. (2008). The Practice of Technology Transfer, PICMET Proceedings, Cape Town, South Africa, Pp. 27-3I.

VERA R., Gerd P., Reigota M. (2007). The second reality: typical problems of Nothern technology transfer experts in the environmental sector in Brazil, Newsletter of the Research Committee 23 Sociology of Science and Technology; International Sociological Association; Issue 5, November; pp. 5-8.

VILLANUEVA Monzón M. (2009). Barreras culturales a la innovación en las pequeñas empresas manufactureras, Fundación Robotiker, Unidad Tecnológica de Madrid, Disponible en: http://www.madrimasd.org/revista/revistal0 /tribuna/tribunas I.asp; [Consultado I3 abril 2009]. 